\title{
El ciberacoso sexual y/o sexista contra las adolescentes. Nuevas versiones online de la opresión patriarcal de las sexualidades y corporalidades femeninas
}

\section{Sexual and sexist cyber-harassment towards young girls. New online versions of patriarchal oppression of female sexuality and corporality}

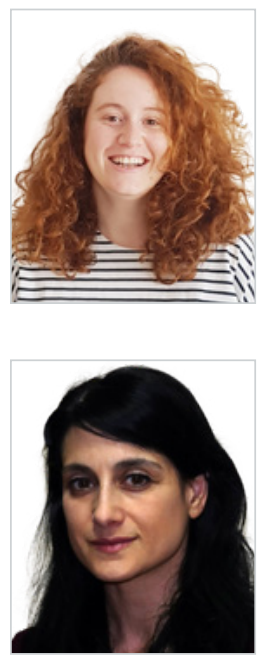

Estibaliz Linares Bahillo. Doctora en el Programa de Derechos Humanos: Retos Éticos, Sociales y Políticos, con la tesis "El Iceberg Digital Machista", y por la que obtuvo la calificación de CUM LAUDE. Graduada en Trabajadora Social, y Máster en Intervención en Violencia contra las Mujeres por la Universidad de Deusto. Investigadora del Equipo Deusto Valores Sociales y coordinadora del Máster en Intervención en Violencia contra las Mujeres de la Universidad de Deusto. Actualmente sus principales líneas de investigación son: género, adolescencia y vida virtual. También colabora como formadora en prevención de violencia entre adolescentes en la Consultoría Sortzen S.l y el Programa Ctrla.

Universidad de Deusto, Bilbao, España

estibaliz.linares@deusto.es

ORCID: 0000-0002-5372-9335

Raquel Royo Prieto. Doctora en Sociología y Diplomada en Trabajo Social. Directora del Máster Universitario en Intervención en Violencia contra las Mujeres de la Universidad de Deusto. Participa en el Programa de Doctorado de la Facultad de Ciencias Sociales y Humanas, en la que también ha impartido las asignaturas Discriminación y violencia de género, y Sociología del género. Miembro del Equipo Deusto Valores Sociales que analiza la Encuesta Europea de Valores. Investigadora principal de diversas investigaciones financiadas por Emakunde-Instituto Vasco de la Mujer y Diputación Foral de Bizkaia. Sus principales líneas de especialización son: maternidades, paternidades y corresponsabilidad, y valores y género.

Universidad de Deusto, Bilbao, España

raquel.royo@deusto.es

ORCID: 0000-0002-9247-5286

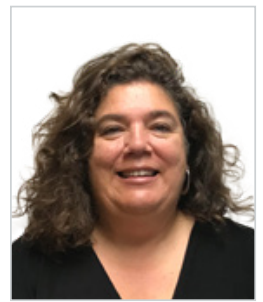

María Silvestre Cabrera. Doctora en Ciencias Políticas y Sociología. Profesora de la Universidad de Deusto. Investigadora Principal del “Equipo Deusto Valores Sociales” que representa a España en la Encuesta Europea de Valores. Ha sido Decana de la Facultad de CCPP y Sociología de la Universidad de Deusto (2004-2009) y Directora del Máster en Intervención en Violencia contra las Mujeres (2003-2009). Ha sido la Directora de Emakunde-Instituto Vasco de la Mujer del Gobierno Vasco (2009-2012). Ha liderado varios proyectos de investigación en convocatorias competitivas y concertadas y ha asumido diferentes cargos de gestión como la Presidencia de la Asociación Vasca de Sociología y la Vicepresidencia de la Federación Española de Sociología. Las principales áreas de especialización son: valores político-sociales y perspectiva de género en las ciencias sociales, áreas en las que posee publicaciones y contribuciones científicas.

Universidad de Deusto, Bilbao, España

maria.silvestre@deusto.es

ORCID: 0000-0002-6928-5126

Cómo citar este artículo:

Linares Bahillo, E.; Royo Prieto, R.; Silvestre Cabrera, M. (2019). El ciberacoso sexual y/o sexista contra las adolescentes. Nuevas versiones online de la opresión patriarcal de las sexualidades y corporalidades femeninas. Doxa Comunicación, 28 , pp. 201-222.

https://doi.org/10.31921/doxacom.n28a11 
El ciberacoso sexual y/o sexista contra las adolescentes. Nuevas versiones online de la opresión...

Recibido: 17/01/2019 - Aceptado: 13/05/2019

\section{Resumen:}

El presente artículo parte de los datos obtenidos en la tesis doctoral "El Iceberg Digital Machista” y utiliza una metodología cualitativa para adentrarse desde una lectura de género y (ciber)feminista en el Ciberacoso sexual y/o sexista que sufren las chicas de la Comunidad Autónoma del País Vasco (CAPV). Para abordar esta realidad, se han realizado 9 grupos de discusión con el alumnado de $4^{\circ}$ de la ESO y $1^{\circ}$ de Bachiller de la CAPV (3 de chicas, 3 de chicos y 3 mixtos), así como 2 foros telemáticos. Este engranaje metodológico cualitativo ha permitido analizar las distintas formas de acoso que sufren las chicas, y profundizar en las estructuras patriarcales/machistas y sexistas que subyacen a estas formas de ciberviolencia.

Entre los resultados más relevantes cabe destacar que, al igual que sucede en la realidad offline, en las redes sociales se reproducen esquemas de género y relaciones asimétricas, y, por ende, violencias machistas. Concretamente, en las redes sociales se dan formas específicas de ciberviolencia contra las chicas, que son más insultadas por su físico, intimidadas sexualmente, y reciben más comentarios sexistas que los chicos.

\section{Palabras clave:}

Ciberacoso sexual y/o sexista; ciberfeminismo; adolescentes; género.
Received: 17/01/2019 - Accepted: 13/05/2019

\section{Abstract:}

This paper, which derives from the PhD thesis "The Macho-driven digital Iceberg", uses a qualitative methodology and gender and (cyber) feminist theory to explore sexual and sexist cyberbullying of female adolescents in the Basque Country. To confront this reality, 9 discussion groups were formed: 3 male, 3 female and 3 mixed, with students from stages equivalent to GCSE and FE (4th year compulsory secondary education and 1st year further education) (Spanish ESO and Bachiller), as well as 2 online forums. This qualitative methodology has allowed us to analyse the different types of bullying suffered by teenage girls, and study in depth the sexist and macho/patriarchal structures underlying these forms of cyberviolence. Amongst the most relevant results, of most significance is that, just as in offline reality, the social media produce schematic and asymmetric gender relationships and, consequently, gender violence. On social media specific forms of cyber violence are aimed at girls, who receive more insults about their physical appearance, are sexually intimidated and receive more sexist comments than boys.

\section{Keywords:}

Sexual/sexist cyber-harassment; cyber feminism; adolescents; gender.

\section{Introducción y contextualización}

Comprender las relaciones humanas en la actual Sociedad de la Información supone descifrar y analizar las interacciones que convergen en los espacios digitales (Castells, 2005). Las Tecnologías de la Relación, Información y Comunicación (de aquí en adelante TRIC) están inexorablemente presentes en las formas de estar, ser y hacer, y así, las formas de información, comunicación y relación han quedado "enganchadas" a estos instrumentos tecnológicos (Instituto de la Mujer, 2008; Zafra, 2010).

Es indudable que las TRIC han traído consigo comodidades, posibilidades y avances tecnológicos, sociales y políticos. Han permitido gestionar un mundo virtual lleno de oportunidades para el desarrollo individual y comunitario. No obstante, este progreso tecnológico, social y cultural, también ha acarreado riesgos digitales marcados por los condicionamientos tradicionales provenientes del mundo offline, como es el caso del sistema patriarcal y de la cultura machista (Zafra, 2005a, 2010; Wajcman, 2004; García y Nuñez, 2008). De este modo, los esquemas de género presentes en las TRIC se han convertido en foco de atención y objeto de estudio de distintas fuentes (Turkle 1997; Alcañiz, 2001; Boix, Fraga y Sedón, 2001; Castaño y Caprile, 2010; Castaño, 2005, 2008; Zafra, 2005a, 2005b, 2010; Díaz, 2001; Wajcman, 2004; Instituto de la Mujer, 2008; Haraway, 1991). 
Paradójicamente, la vida virtual se sumerge en una ambivalencia constante derivada de la coexistencia de espacios sumamente subversivos para la ruptura de los esquemas de género y la exploración de identidades no-normativas ${ }^{1}$ (Haraway, 1991; Plant, 1998; Turkle, 1997; Zafra, 2005b); con otros marcados por uno de los mecanismos patriarcales más eficaces para el sustento de la dominación masculina: la violencia contra las mujeres (Lagarde, 1990). Así, en aras de demostrar cómo en el mundo virtual se reproducen formas concretas de dominación patriarcal, diversos estudios emergentes se están centrando en las ciberviolencia(s) que sufren las mujeres (Gobierno Vasco, 2013; Megias y Ballesteros, 2014; EIGE, 2018; UNESCO, 2017; Lenhart 2009; Powell y Henry, 2014; Strassberg, et al. 2012; Delegación del Gobierno para la Violencia de Género, 2014; Navarro, 2016; EIGE, 2018).

Algunas de estas investigaciones señalan que el porcentaje de las chicas que sufren acoso a través de las TRIC supera al de los chicos $-9 \%$ versus $6 \%$, respectivamente, según el Instituto Europeo de la Igualdad de Género (EIGE, 2018); y $12 \%$ versus 8\%, según UNESCO (2017)-. Sin embargo, la evidencia empírica muestra que el problema es más estructural y cualitativo, que cuantitativo, dadas las diferencias de género existentes en el objeto del acoso y en las formas que este adopta. En este sentido, las chicas son objeto de diversas modalidades de ciberacoso sexual y/o sexista, según sea el caso, lo que genera espacios intimidatorios contra sus cuerpos y su sexualidad (Lenhart 2009; Powell y Henry, 2014; Strassberg et al., 2012; Delegación del Gobierno para la Violencia de Género, 2014; Navarro, 2016; EIGE, 2018).

Nuestro propósito es explorar estas formas de agresión en una de las etapas vitales más cruciales para la configuración de la identidad: la adolescencia (Jiménez-Albiar et al., 2012; Vázquez, Estébanez y Herbón, 2013; Tubert, 2008; Pineda y Aliño, 1999; Rovira, 2001; Frosh, Phoenix y Pattman, 2002: 67; Martino y Pallota-Chiarolli, 2005; Renold, 2002, 2007; Egan y Hawkes, 2012). En concreto, este artículo quiere adentrarse en el ciberacoso sexual y/o sexista que sufren las chicas de la CAPV desde una perspectiva cualitativa, de género y (ciber)feminista. Para ello, en coherencia con los resultados de las investigaciones revisadas, se parte de dos hipótesis principales: 1) las chicas sufren formas de ciberviolencia concretas, como la sexual y sexista; y 2) estas formas de ciberviolencia se sustentan en un legado patriarcal y machista ${ }^{2}$.

Desde estas premisas, el artículo, primero, recoge algunas de las investigaciones previas sobre las formas de violencia contra las mujeres que se están reproduciendo en la Red entre las personas adolescentes y revisa el marco conceptual utilizado en este ámbito, optando por un término acorde con la perspectiva ciberfeminista, entendida como una estrategia reflexiva, crítica y política diversa que busca el empoderamiento de las mujeres en el mundo digital, y a la vez, la subversión y la transgresión de los condicionamientos tradicionales (Zafra, 2005b; Wajcman, 2004; Haraway, 1991; Turkle, 1991; Paasonen, 2011). Este primer análisis bibliográfico sustenta la parte empírica, que nos sumerge en los discursos de personas adolescentes de 15 a 17 años, de $4^{\circ}$ de la ESO y $1^{\circ}$ de Bachiller de 9 institutos diferentes de la CAPV y nos permite analizar las distintas formas de acoso que sufren las chicas, así como profundizar en las estructuras patriarcales/machistas y sexistas que subyacen a estas formas de ciberviolencia, y conocer sus consecuencias.

1 Las personas con identidades no-normativas subvierten la normatividad (hetero)patriarcal, bien porque sus comportamientos rompen con los mandatos de género, bien porque su orientación sexual no encaja con los esquemas heterosexistas (Valdes, 2013).

2 Este artículo parte de los resultados más relevantes obtenidos en la Tesis Iceberg Digital Machista. 
Con estas inquietudes, el presente artículo no se redacta con un propósito generalizador, sino exploratorio, tratando de generar espacios de análisis y debate, que permitan visibilizar y ahondar en los mecanismos patriarcales que están incidiendo en la Red. Es, pues, una propuesta subversiva que busca indagar en la reproducción de desigualdades que tienen lugar en un mundo que se pronosticaba infinito para la búsqueda de identidades y la legitimación de relaciones horizontales.

\section{2. (Ciber)violencias entre adolescentes}

La violencia ejercida por iguales encuentra nuevos escenarios como la Red. Así, diversas fuentes centran sus esfuerzos en comprender y analizar lo que ya lleva tiempo denominándose como cyberbullying (Bartrina, 2014; Buelga y Pons, 2011:92; Buelga, Cava y Musitu, 2010; Delegación del Gobierno para la Violencia de Género, 2014:25-26; Del Rey, Felipe y Ortega-Ruiz, 2012: 17; Mitchell et al., 2016). Este ejercicio de violencia se produce especialmente entre el alumnado, y, aunque guarda semejanzas con el acoso y el bullying tradicional -particularmente en sus orígenes y causas- presenta rasgos específicos que potencian aún más, si cabe, la agresión recibida y percibida. Estas características propias se pueden resumir en tres vertientes: 1) el anonimato del agresor, que posibilita una mayor impunidad, y con ella, un mayor daño y una mayor situación de indefensión; 2) las agresiones pueden sucederse permanentemente, muy rápido y ejercerse por un gran número de personas a la vez e instantáneamente; y 3) el acoso tiene lugar ante una gran "audiencia” y con gran difusión.

Entre los estudios sobre cyberbullying, están los que afirman que no existen diferencias relevantes en función del género (aunque tampoco integran un análisis profundo desde esta perspectiva) (Buelga y Pons, 2011:92; Buelga, Cava y Musitu, 2010; Del Rey, Felipe y Ortega-Ruiz, 2012; Mitchell et al., 2016); y los que concluyen que, si bien tanto chicas como chicos sufren cyberbullyig, las primeras sufren formas concretas de dominación, relacionadas con la agresión de su corporalidad y sexualidad (Bartrina, 2014; Gobierno Vasco, 2013; Delegación del Gobierno para la Violencia de Género, 2014; Lenhart, 2009; Powell y Henry, 2014; Strassberg et al., 2012; UNESCO, 2017; Navarro, 2016; EIGE, 2018).

Dadas las características propias del ciberbullying, diferentes estudios comenzaron a indagar, analizar y utilizar un término propio para referirse al envío de imágenes y videos de contenido sexual y erótico: el sexting (Lenhart 2009; Strassberg et al., 2012; Delegación del Gobierno para la Violencia de Género, 2014; Powell y Henry, 2014). Sin embargo, mientras para algunas fuentes el sexting supone la acción de enviar mensajes eróticos que contienen fotos y/o videos -un juego erótico-; para otras el término se refiere al envío de fotos y/o videos de una chica con una intención de dominación y extorsión. Esta divergencia terminológica genera un problema conceptual y legal, y no permite dirimir si se atiende a una agresión, o no.

Por ello, Powell y Henry (2014) proponen una distinción conceptual dentro de este tipo de actos, entre el acoso (acto criminal con la intención de intimidar), al que denominan como sextorsión, y la experimentación (un juego sexual entre distintas personas), a la que llaman sexting. Esta diferencia ayudaría a precisar el significado del sexting, que sería el envío de imágenes y/o fotos con autorización.

A pesar de estos esfuerzos por delimitar estas formas de agresión, y de la constatación empírica de que son las chicas quienes las sufren de una manera desproporcionada, los estudios mencionados no ahondan en un análisis cualitativo y profundo de las estructuras y mecanismos que subyacen a esta realidad. Su comprensión requiere transversalizar la perspectiva de género, ya que como alegan distintas fuentes (Egan y Hawkes, 2012; Martino y Pallota-Chiarolli, 2005: 99; Renold, 2002, 
2007; Ringrose y Renold, 2010), los esquemas de género instauran relaciones de poder y posiciones asimétricas que sitúan a la "masculinidad hegemónica"3 en la cúspide del "orden social", de forma que toda persona que rompe y/o escapa de lo normativo queda estigmatizada (desde chicos que muestran ser sensibles, estudiosos u homosexuales, hasta cualquier chica que cuestione los mandatos de género, como exponer su sexualidad). Esta asimetría, legitimada y normalizada -tal y como Lagarde (1990) ${ }^{4}$ y De Beauvoir (1945) explican-, sustenta mecanismos concretos de poder y opresión contra las mujeres, como la violencia simbólica y explícita, o la opresión de la sexualidad femenina como dispositivo de control social.

Estas teorías constituyen bases teóricas relevantes para entender estas formas de violencia y sus causas intersubjetivas, lo que permite evitar discursos culpabilizadores y términos que no dan cuenta del verdadero carácter del fenómeno estudiado. Por estos motivos, a continuación, se procede a elaborar y argumentar una terminología propia que introduzca y tenga como eje transversal la perspectiva de género y la lectura (ciber)feminista.

\section{3. "Lo que no se nombra, no existe". En búsqueda de una terminología que visibilice el origen de las (ciber)violencias que sufren las jóvenes}

En primer lugar, a partir de la revisión bibliográfica realizada sobre la conceptualización de la violencia en el marco cibernético (Gobierno para la Violencia de Género, 2014; Buelga, Cava y Musitu, 2010; Buelga y Pons, 2011; Del Rey, Felipe y Ortega-Ruiz, 2012; Mitchell et al., 2016; Bartrina, 2014; Lenhart 2009; Strassberg, et al., 2012; Powell y Henry, 2014), se propone el término ciberacoso, dado que es un concepto global -no anglosajón-, y que se refiere a conductas realizadas en este ámbito que dan lugar a una relación asimétrica, en la que una persona ocupa una posición dominante, y hace que la otra asuma un rol de sumisión (Delegación del Gobierno para la Violencia de Género, 2014).

En segundo lugar, al abordar una terminología concreta sobre el acoso que sufren las chicas jóvenes -que abarque los acosos anteriormente mencionados-, una de las pocas propuestas terminológicas encontradas es la establecida por la Delegación del Gobierno para la Violencia de Género (2014). Esta define el ciberacoso por razón de violencia de género como:

Aquellos comportamientos que, utilizando las TIC, tienen como objetivo la dominación, la discriminación y, en definitiva, el abuso de la posición de poder donde el hombre acosador tiene o ha tenido alguna relación afectiva o de pareja con la mujer acosada. Igualmente, este acoso debe ser repetitivo, no consentido, debe suponer una intromisión en la vida privada de la víctima y, el motivo de dicho acoso, debe estar relacionado en alguna medida con la relación afectiva que tienen o tuvieron acosador y acosada (Delegación del Gobierno para la Violencia de Género, 2014:27).

3 Estas explicaciones se estructuran a partir de la noción teórica de Connell (1995), que sostiene que las construcciones de género no son entes monolíticos, sino que deben comprenderse en su contexto sociocultural y económico, lo que da lugar a diferentes configuraciones según la cultura imperante. No obstante, la cultura occidental prescribe una "masculinidad hegemónica" -vinculada al hombre blanco, heterosexual, autoritario, con fuerza física y sexualmente activo- que ocupa el lugar preponderante en el orden de género. Esta domina a otras masculinidades subordinadas que rompen con estas normas y a cualquiera de las feminidades.

4 Lagarde (1990) y De Beauvoir (1945) entienden que la construcción femenina ha sido socialmente oprimida y dañada por la violencia estructural impuesta utilizando medios muy diversos como la subordinación de la sexualidad de las mujeres, la cosificación de sus cuerpos, la ideología de la maternidad intensiva, las violencias físicas... Asimismo, el sistema patriarcal ha otorgado atributos concretos a la feminidad como la dependencia de la mirada ajena (especialmente de la masculina), que ha sustentado una rivalidad femenina, así como una dicotomía entre "buenas" -o feminidades recalcadas como establecería Connell (1995)- y "malas" mujeres, que establece una distinción entre los arquetipos femeninos más próximos a la normatividad patriarcal, y los alejados de esta, que son estigmatizados y condenados socialmente. 
La aproximación y la visibilización que realiza el estudio de la Delegación del Gobierno para la Violencia de Género sobre las formas específicas de ciberviolencia que se dan contra las mujeres, se considera particularmente interesante. No obstante, se discrepa en que el ciberacoso o la ciberviolencia tenga que producirse únicamente en el marco de la pareja o de una relación afectiva-sexual. Por ello, teniendo en cuenta la aportación de los estudios sobre la violencia sexual (Crouh, 2001; Mora, 2004; Osborne, 2001, 2009; Weeks, 1993) y considerando, a su vez, que se quiere utilizar una terminología cercana al lenguaje cotidiano, se opta por realizar una aproximación conceptual al acoso sexual -en su versión tradicional-, para así, poder analizar si este término corresponde con el interés del presente estudio.

En la definición y delimitación del "acoso sexual” nos enfrentamos a retos y limitaciones. No existe un consenso terminológico, sino que se usan nociones dispares como "acoso por razón de sexo", "acoso sexual" o "acoso sexista". Los estudios y leyes vigentes que se ocupan de la definición y/o sanción de estas conductas no están exentas de esta confusión terminológica. La Ley Orgánica 3/2007, de 22 de marzo, para la igualdad efectiva de mujeres y hombres, en su artículo séptimo expone lo que entiende por acoso sexual y/o por razón de sexo, equiparando ambos términos. Asimismo, Gobierno Vasco (2011:3), Osborne (2001, 2009), Weeks (1993), Renold (2002), Mora (2004) y Crouh (2001) entienden que el acoso es un elemento regulador de conductas que obliga a las mujeres a la contención y la invisibilidad de su sexualidad, mientras que legitima y normaliza la dominación sexual masculina.

Sin embargo, en el análisis de los textos antes citados conviene establecer una distinción conceptual entre los términos mencionados. El acoso sexista se refiere a mensajes y actitudes dirigidos a la mujer -que esta oye, lee, nota, palpa...- en función de su condición de género (que le atribuye una serie de características inferiores a las del hombre). El acoso sexual, por su parte, se define como cualquier comportamiento, verbal o físico, denigrante con fines sexuales. Además, ambos acosos pueden suceder en cualquier situación de la vida cotidiana -no únicamente en el ámbito laboral (como se ha recogido en algunas leyes)-, y constituyen actos concretos que posibilitan la asimetría de poderes, y la discriminación por razón de género en cualquier contexto, sustentando "el orden social de las cosas".

En definitiva, tanto el acoso sexista como el acoso sexual son formas de hacer referencia al sexismo y a la política sexual en la que hombres y mujeres ocupan lugares asimétricos. Por tanto, no corresponden a una única forma de violencia o a un tipo de agresión concreta, y requieren de una visión holística que los comprenda como múltiples conductas que pueden presentarse potencialmente en cualquier escenario de interacción social entre los géneros (Mora, 2004:3; Osborne, 2001, 2009; Weeks, 1993).

Teniendo en cuenta las aportaciones conceptuales revisadas, este artículo propone como términos de referencia para dar cobertura a la realidad objeto de estudio, los términos de ciberacoso sexista y ciberacoso sexual. El primero abarcaría actitudes, verbalizaciones o comportamientos que se produzcan en las TRIC y que se sustenten en estereotipos de género; por ejemplo, agresiones dirigidas a la imagen corporal, insultos, comentarios o imágenes que reproducen estereotipos de género (como las imágenes sexistas que se puedan colgar en alguna red social y que denigren a la mujer, por su condición de sexo).

El segundo término acuñado en esta investigación es el ciberacoso sexual, acotado como todas aquellas actitudes, verbalizaciones y comportamientos de naturaleza sexual que se produzcan en las TRIC y que tengan el efecto de atentar contra la 
dignidad de una chica, en particular cuando se crea un entorno intimidatorio, degradante u ofensivo; por ejemplo, el envío y difusión de fotos y/o videos de su intimidad sin el consentimiento de la joven.

No obstante, como señalan los estudios realizados por la Delegación del Gobierno para la Violencia de Género (2014) y Ruiz (2014), resulta difícil delimitar una lista cerrada de acosos y ataques, debido a múltiples factores, entre ellos: las diferentes formas de expresar y utilizar las tecnologías; el propio dinamismo tecnológico, con el que se desarrollan nuevas estrategias de acoso y agresión; y el hecho de que las propias adolescentes no detectan y normalizan las agresiones, sin denunciarlas. Por tanto, la investigación empírica es una vía que nos permite reconocer estas formas de agresión y/u otras que quizá los estudios elaborados hasta ahora no habían podido detectar, pudiendo enriquecer el estado de la cuestión, teniendo siempre presente que el artículo carece de pretensiones de extrapolación y se inscribe en el contexto de la CAPV.

\section{Metodología para recoger las voces de los y las nativas digitales}

Como se mencionaba anteriormente, este artículo pretende adentrarse desde una lectura de género y (ciber)feminista en el ciberacoso sexual y/o sexista que sufren las chicas de la CAPV, partiendo de las siguientes hipótesis:

- H1- Las chicas sufren formas de ciberviolencia concreta, como el (ciber)acoso sexual y/o sexista a través de las redes sociales.

- H2- Estas formas de ciberviolencia se enraízan en un legado patriarcal y machista.

Para ello, se utiliza una metodología cualitativa, ya que como señalan Taylor y Bogdan (2000:19) y Ruiz Olabuénaga (2012: 44), esta nos permite estudiar la dimensión subjetiva, adentrarnos en las palabras, creencias y opiniones, y conocer en su esencia los significados intersubjetivos del hecho que quiere ser estudiado. Desde esta perspectiva, se acotan los siguientes objetivos:

1. Analizar las distintas formas de acoso que sufren las chicas a través de los discursos de las personas adolescentes que participan.

2. Profundizar en las estructuras patriarcales/machistas y sexistas que subyacen a estas formas de ciberacoso sexual y/o sexista.

3. Conocer las consecuencias que acarrean estas formas de ciberviolencia.

Para el abordaje de estos objetivos, se opta por las técnicas del grupo de discusión y el foro telemático. Ambas se retroalimentan, y desde la intrasubjetividad de las personas adolescentes que participan, se consigue explorar de forma rica y complementaria los intereses planteados. En concreto, la primera técnica ayuda a crear espacios de diálogo e interrelación (Ibañez, 2010; Gutiérrez, 2008). Esto facilita la comprensión del imaginario cultural de las personas adolescentes participantes, así como de las razones e ideologías que sustentan las situaciones de ciberacoso sexual y/o sexista.

La segunda técnica, el foro telemático, se selecciona por ser una herramienta informática que permite explorar el discurso colectivo dentro de la Red y se asemeja a lo que viven, escriben, leen y comparten las personas adolescentes (Hooley, Marriott y Wellens, 2013; Chriss y Stewart, 2000). No obstante, se asume como una técnica complementaria y enriquecedora 
del grupo de discusión, y que posibilita la generación de discursos y diálogos fluidos, flexibles y sin encorsetamientos, a partir de la utilización de fotos, comentarios, videos...

Para organizar la participación en ambos procesos, primero, se establece el contacto con 9 institutos diferentes ${ }^{5}$ pertenecientes a la CAPV (3 por cada territorio histórico) ${ }^{6}$, y se marcan tres criterios principales: 1) que el grupo esté integrado por un número de personas que oscile entre 7 y 12 por instituto; 2) que sea alumnado perteneciente a los cursos de $4^{\circ}$ de la ESO y $1^{\circ}$ de Bachiller (es decir, que tengan entre 14 y 17 años); y 3) que en cada territorio histórico exista al menos un grupo de chicas, uno de chicos, y otro mixto ${ }^{7}$.

Una vez realizados los grupos de discusión, se anima a las personas que los integraron a formar parte de los foros telemáticos. De las 83 personas participantes en la investigación cualitativa (42 chicas y 41 chicos), fueron 17 chicas y 17 chicos quienes participaron en los dos foros telemáticos (uno de chicas y otro de chicos). El número de componentes de los foros es más reducido ya que, como precisan Hesse-Biber y Leavy (2011), al ser una técnica interpretativa, su propósito, la profundización en vivencias y experiencias, no requiere de una muestra tan amplia. La organización de los grupos se gestionó a través del centro escolar.

\section{El ciberacoso sexual y/o sexista que sufren las adolescentes a través de los discursos de los y las nativas digitales de la CAPV}

Las páginas siguientes nos sumergen en el discurso y en el hipertexto producido por las personas adolescentes que han participado en la investigación. En primer lugar, se presentan los principales resultados de los grupos de discusión y, en un segundo momento, se exponen algunos de los elementos particularmente relevantes de los foros telemáticos. Los resultados de los grupos de discusión nos acercan a las características comunes del ciberacoso sexual y/o sexista que sufren las chicas, a su percepción y vivencia, y también a las ciberviolencias ejercidas contra las personas que transgreden la heteronormatividad. Los foros telemáticos complementan y refuerzan los resultados de los grupos de discusión, ahondando en algunos elementos claves de las formas de ciberviolencia ejercidas contra las chicas participantes.

5 Como se ha señalado anteriormente, el presente artículo nace de la tesis doctoral "El Iceberg Digital Machista”, cuyo engranaje metodológico es más complejo, y comienza con una primera fase de cuestionarios cerrados que da paso a estos grupos de discusión. Para ello, en la primera fase, se pide al alumnado interesado en participar en los grupos que se inscriba, lo que da lugar a un total de 190 chicas y 147 chicos inscritos, entre quienes se seleccionan las y los participantes de los grupos, en base a los criterios mencionados.

6 Para garantizar los criterios éticos de la investigación se realizan diferentes procesos como enviar al instituto una "carta-modelo" que informa del estudio, y posteriormente una carta de autorización de la participación de los y las adolescentes para la persona responsable legal. El instituto se encarga de enviar las cartas de autorización, así como de elegir el aula y el lugar de realización de los grupos.

7 Este tercer criterio se propone dada la importancia que adquiere la identidad en esta etapa, tal y como sostienen Frosh, Phoenix y Pattman (2002). Esto es, la división de estos espacios también nos otorga elementos de análisis sobre los esquemas de género. Cabe precisar que, con intención de salvaguardar el anonimato, se asigna a cada grupo un número para su codificación y a cada participante, un código alfanumérico en relación al grupo de pertenencia. 


\subsection{Rasgos comunes y proceso del ciberacoso sexual y/o sexista}

Según las personas que participan en los grupos, las situaciones de ciberacoso sexual y/o sexista se están dando con una frecuencia alta en su contexto cercano. De hecho, en 8 de los 9 grupos realizados han existido uno o varios casos de estos tipos de acoso. Veamos algunos ejemplos concretos:

D10 (chica, 16 años)- Es una chica que no solo le mandó fotos a uno, sino a varios, y eran sus amigos. Uno le mandó a otro, y el otro al otro...

H9 (chico, 16 años)- Un chico tenía una foto y lo subió a su Instagram y que, supuestamente, lo subió sin querer, pero era la foto de la chica desnuda y pronto lo tenía todo el mundo... Le habló la chica, lo borró... (...) Lo pasó mal, mal... Los primeros días tú veías cinco corros en el instituto y en los cinco corros, la misma foto. Y veías a la chavala sola por

el patio... Porque ya sabía lo que había...

Asimismo, a través de los discursos de las personas adolescentes, se puede constatar que el ciberacoso sexual y/ o sexista se produce también en el marco de parejas no heterosexuales, así como en relaciones esporádicas o puntuales (como relaciones de una noche, las que se gestan por Internet...).

El análisis de las narraciones de las personas adolescentes consultadas, permite concluir que ambos tipos de agresión interactúan y presentan rasgos comunes que se plasman en la reproducción de un mismo itinerario. Estos hallazgos se recogen en el siguiente esquema:

Ilustración 1. Características comunes y proceso identificado en los casos de ciberacoso sexual y/o sexista

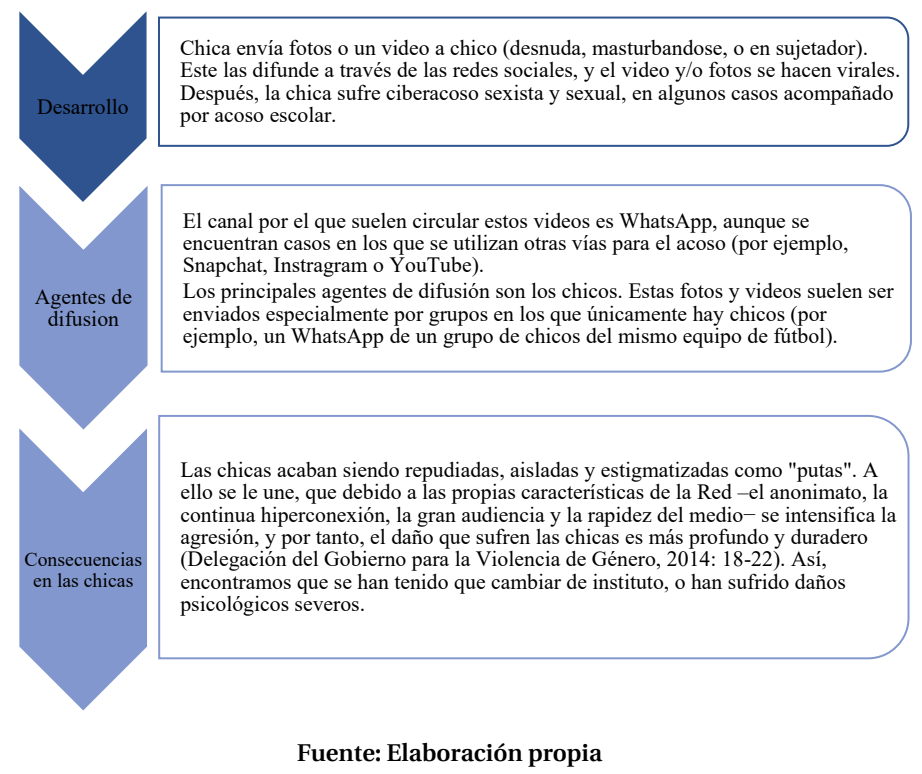


El ciberacoso sexual y/o sexista contra las adolescentes. Nuevas versiones online de la opresión...

\subsection{El ciberacoso sexual y/o sexista, prácticas normalizadas en la masculinidad}

Como se puede apreciar en la ilustración anterior, en los grupos de discusión se constata que los chicos son los principales agentes de difusión de este tipo de contenidos. De hecho, especialmente en aquellos grupos formados únicamente por chicos, se observa que existe una mayor normalización de estos actos. En este sentido, los chicos se expresan con naturalidad sobre aquellos vídeos y fotos que se envían de chicas desnudas que no conocen o que tienen contenido pornográfico. He aquí algunos ejemplos de lo señalado:

H1 (chico, 16 años)- Porque se venden más fácil. A una chica le pasan una foto de un chico en pelotas y yo creo que no tienen tendencia a mandarlo o enviarlo... Pues lo ha visto y ya está, pero un chico es: "buah, ¡mira lo que me han mandado!”... Y se lo pasan a uno, se lo pasan al otro... Y se la suda...

B10 (chico, 16 años)- Es que me imagino que muchos chicos pensarían: “ibuah, esta envía fotos!”, pues le hablan todos a saco.

También sus compañeras se refieren a que ellos son los principales agentes de difusión y agresores. En referencia a ello, se destaca la siguiente cita:

D10 (Chica, 16 años)- Y no dicen en plan... que yo ya he escuchado a los chicos en plan: “ibuah, qué guarra!”, es lo que más he escuchado decir siempre, ¡qué guarra!...

B1O (Chico, 16 años) -Pues, por eso básicamente, porque dicen: "buah, esta es la pedazo de guarra, la hablo y mañana quedo con ella". Y ya está.

La visualización habitual de contenidos pornográficos, heterodirigidos a la satisfacción de los deseos masculinos de objetivización y erotización del cuerpo femenino -videojuegos, anuncios, pornografía heterodirigida...-, y la educación en códigos de libertad sexual y en la necesidad adquirida de exposición de "grandeza", favorece que los chicos adopten estas posturas (Martino y Pallota-Chiarolli, 2005; Ringrose y Renold, 2010; Zafra, 2005a; Gil, 2008; Vázquez, Estébanez y Herbón, 2013). El consumo elevado de este contenido merma la actitud crítica y sustenta y normaliza los actos que hemos descrito (Zafra, 2005a). Así, tal y como Vázquez, Estébanez y Herbón (2013) apuntaban, todas estas diferencias y desigualdades se siguen manteniendo debido a esta normalización e invisibilización que se produce gracias a la sumersión continua en esta información sexista y machista.

\subsection{La normatividad de género en las formas de ciberacoso}

Las personas que han formado parte de los grupos de discusión señalan cómo en las situaciones anteriormente descritas una audiencia masiva se convierte en juez únicamente de la actitud de una joven. Incluso en aquellos casos en los que se han grabado manteniendo relaciones sexuales, y en escena aparecen chica y chico, es ella la que es condenada y estigmatizada, mientras que al chico se valora como un héroe y se le felicita por la "gran hazaña". Así lo manifiestan los y las adolescentes: 
F6 (chica, 16 años)- A un chico le tratan como a un héroe, y a una chica le tratan como a eso...

B3 (chico, 17 años)- Eso, mucha gente, incluso, en vez de insultar al tío, le darían como ánimos; en cambio, la chica es una guarra.

B10 (chico, 17 años)- La tía es una guarra porque está follando con un tío en la calle. Al final todos pensamos que ella es un poco suelta....

Todo ello muestra que la sexualidad femenina queda definida por parámetros coercitivos de sumisión y pasividad, y en caso de romperlos, la construcción de "mala mujer" se cierne sobre ellas, que ya no son vistas como "mujeres", ni tan siquiera como personas (De Beauvoir, 1949; Lagarde, 1990; Megias y Ballesteros, 2014).

Debido a la construcción de "mala mujer", chicas y chicos -pero sobre todo estas- responsabilizan únicamente a las chicas de estos casos, y no empatizan con ellas. De hecho, en varios grupos una de las primeras palabras que mencionan ante un caso de ciberacoso es "tonta". He aquí algunos ejemplos de lo señalado:

D4 (chica, 15 años)- Que ha sido un poco tonta (por mandar la foto).

A6 (chica, 15 años)-Yo opino que la chica ha sido tonta, por mandar la foto, porque si quieres que te vea desnuda vas a su casa y te desnudas, y punto.

F4 (chica, 16 años)- Primero, que la chica no debería haber mandado las fotos. Bueno, yo creo que eso no sé quién lo podría hacer... porque sabes que las fotos que envías corres un riesgo, y por seguridad...

G2 (chica, 16 años)- Primero, que la chica no lo tenía que haber hecho. Tonta por haberlo hecho y confiar... Es que puede ser tu idea que era tu novio y querías casarte con él, pero no... Tus cosas íntimas y, más tus fotos, con nadie.

Comprenden el problema desde un prisma individualista, y no holístico y colectivo. De hecho, solo una chica (I7) se plantea la posibilidad de que exista una responsabilidad colectiva al señalar que son únicamente las chicas quienes sufren estos agravios por el simple hecho de serlo, visibilizando asíla construcción machista que subyace a estos actos. Esta perspectiva le permite empatizar con una chica que se encuentre en este tipo de situaciones, tal y como muestran sus manifestaciones: "Ella ya tiene una responsabilidad, pero si confía en él... Es como si yo estoy con mi pareja, y no pienso en ningún momento en que se la pueda enviar (la foto) a otra persona" (I7, chica 15 años).

Asimismo, se encuentran ejemplos claros de las actitudes de pasividad y contención que tienen sobre su propia sexualidad, que contribuyen a que contemplen este tipo de situaciones como un acto privado. En varios grupos se señala que en el caso de querer "enseñar" algo, que sea en persona o de forma íntima; y algunas chicas sostienen que aquellas que envían estos vídeos o fotos desean llamar la atención, tal y como se puede leer en los siguientes comentarios:

F4 (chica, 16 años)- Yo creo que la chavala iba buscando eso... A ver, son fotos como de un anuncio, y al final, esas te las haces para que te vean...

F3 (chica, 16 años)-Vamos, lo que quería era más que nada llamar la atención.

H1 (chico, 16 años)- Y yo creo que las chicas cuando se creen que las quieren son más ingenuas, mientras yo creo que los chicos llegan a un punto que siempre son precavidos, yo creo que tienen un límite... En cambio, la chica, no lo tengo tan claro. 
Las chicas se reconocen como víctimas y sexualmente pasivas (Beauvoir, 1949:467), y exigen a otras que cumplan con este mandato. La asunción de su cosificación les impide empatizar con otras chicas, y hace que incluso quieran alejarse de ellas, repudiándolas y estigmatizándolas (Egan y Hawkes, 2012; Martino y Pallota-Chiarolli, 2005: 99; Renold, 2002, 2007). Marcan una clara diferencia entre ellas, "las buenas" - que nunca difundirían una foto-, y las otras, "las malas", como indica una de ellas: "Yo creo que un grave problema también es que nos los decimos entre nosotras" (F7). A continuación se presentan algunas expresiones que ejemplifican lo señalado anteriormente:

D10 (chica, 16 años)- Que ha sido un poco guarra

A3 (chica, 16 años)- No sé, a mí me da asco.

A5 (chica, 15 años)- ¡Que le gustaba llamar la atención!

F5 (chica, 16 años)- Es que, ¿sabes lo qué pasa? Que esa gente se cree que se habla muchísimo de ellas, y es que no es así...

Por otra parte, algunas chicas y chicos (A8, B10, B3, H1, H3), con el fin de argumentar por qué se crea este tipo de ciberacoso, aluden a uno de los estereotipos adscritos a la feminidad: "la debilidad". Así, señalan que estas chicas son "más manipulables", "más ingenuas" o "cogen confianza muy rápido". Observamos que, de nuevo, se atribuye una causa individual a un problema cuyo origen es marcadamente social.

\subsection{Los cuerpos de las adolescentes como territorios de dominación patriarcal}

Los cuerpos de las chicas se convierten también en territorios de dominación. En los discursos de las chicas y los chicos se han encontrado otros ejemplos de ciberacoso sexista y sexual que se reproducen en las redes. Como recogía el estudio de Gobierno Vasco (2013), las chicas, simplemente por el hecho de serlo, reciben más manifestaciones despectivas sobre su aspecto físico, así como más comentarios de carácter íntimo y sexual a través de las redes sociales. Los siguientes relatos ejemplifican lo señalado:

Al (chica, 15 años)- Luego hay cosas, que yo he ignorado, preguntas porque la gente no tenía que saber, ni... hay preguntas de si he follado con alguien, o cosas que la gente no tiene que saberlas.

F2 (chica, 16 años)- Es que, es que... te ponen por ejemplo: “ichúpame la polla!”, o yo que sé... y entonces coges y respondes una tontería... pero es que, al final, ya te están faltado al respeto...

F4 (chica, 16 años)-Y te hacen preguntas súper íntimas...

Estas manifestaciones en concreto se están produciendo a través de un canal que hasta ahora no había sido nombrado: Ask $^{8}$. Como ejemplo, encontramos el "ranking" de chicas que se menciona en los distintos grupos y foros, en el que se añade el nombre de una chica y un grupo de personas anónimas la puntúa, y en ocasiones incluye comentarios sexistas contra ella.

8 Esta Red funciona como un consultorio de preguntas, en la que de forma anónima o no -esto lo elige cada persona-, se lanza una pregunta en la Red, y las personas que están conectadas deben contestar. 
Tal y como se detecta en los grupos, esta objetivación de las jóvenes, a la que se suma el valor que estas otorgan a su cuerpo en la definición de su autoconcepto, genera más sufrimiento en ellas que en ellos. De forma general, para las chicas consultadas esta Red se ha convertido en un lugar dañino y tedioso, y como ellas mismas explican, la encuentran "asquerosa". Así lo expresan las chicas del grupo 6:

F4 (chica, 16 años)- Tú pones una nota del 0 al 10, entonces a mí eso es lo que me parece que humilla a la gente, y por otro te sientes como que la gente estuviera por encima de otra, a mí eso me parece fatal, es que hay chavalas a las que les puede hacer mucho daño.

F7 (chica, 16 años)- Sí, yo por ejemplo cuando vi mi nota, y me quedé un poco así, y pensé: “iyo soy un 6?” Y te quedas como igual eres un 6, pero luego lo piensas y dices: "él es un 6". Es que yo no soy un número, yo soy mucho más que un número... A mí no me pongas un número...

\subsection{Las ciberviolencias contra las personas que subvierten la heteronormatividad}

Como señalan Philips (2013) y Ringrose y Renold (2010), es necesario visibilizar las actitudes de LGTBI-fobia que también emergen en la Red. Esta cultura machista que configura posiciones y normas de género, genera también que aquel o aquella que transgreda los mandatos heteronormativos sea objeto de una condena social. Estas formas de violencia también suelen encontrarse en las conexiones en directo que se realizan a la hora de jugar online, reproduciendo las "crueldades normativas" (Ringrose y Renold, 2010). En palabras de dos chicas:

I5 (chica, 15 años) - Hay un chico en YouTube que hace un poco de todo, y la gente le pregunta a ver si es gay y así... Solo por el hecho de... No porque él lo sea ni nada, que habla de cosas, no sé cómo decirte, normales... Y la gente se piensa que es gay.

E3- Yo conozco a una chica, pero que antes era chico, y ahora es chica, y que sufrió acoso en el colegio, pero también a través de las redes sociales. Ella jugaba en un equipo profesional y la gente se metía mucho con la chica, o sea, siempre en los chats y en estas cosas la decían cosas malas, y solo por ser chica y haberse cambiado de sexo.

Como se refleja en estos relatos, la Red se convierte en un espacio desigual y asimétrico, que configura posturas jerarquizadas, y la heteronorma patriarcal actúa como telón de fondo en el que se desarrollan las interacciones de las y los adolescentes participantes. Por ello, se considera sumamente necesario profundizar en el estudio de este tipo de agresiones en investigaciones ulteriores.

El análisis de los discursos obtenidos en los grupos de discusión, abordado en los apartados precedentes, se complementa con el del hipertexto de los y las adolescentes que han participado en los foros telemáticos. A continuación se presentan los resultados más destacados de dichos foros.

\subsection{Ciberviolencias contra las chicas como ejercicio de dominación}

Como sucedía en los grupos de discusión, los foros evidencian que en la Red se dan formas específicas de violencia contra las mujeres, y que sus corporalidades se convierten en objetos de agresión. En estos espacios, tal y como sostienen Bour- 
dieu (1949), Osborne (2001, 2009), Weeks (1993) y Lagarde (1990), la violencia contra las mujeres constituye un mecanismo de control y mantenimiento del "orden social”.

En este sentido, las personas que participan en los foros manifiestan que la violencia es generalizada y que incluye prácticas que han sufrido mujeres famosas o anónimas, cuyas consecuencias coinciden en ambos casos: estigmatización y ciberacoso sexista y sexual contra la mujer. Además, también se refleja que son especialmente los chicos los que reciben casi semanalmente -principalmente en los grupos de WhatsApp compuestos únicamente por chicos- videos y fotos de chicas. En palabras de dos chicos que han participado en los foros:

por The Mannis el Jue 13 Oct 2016 - 23:03

A todos nos han llegado fotos, videos... De tías de gente desnuda o, no? Solo hay que ver lo que ha pasado con los futbolistas del Eibar... De un día para el otro todos teníamos ese video, todos los comentábamos

por MIRTXI el Miér 28 Sep 2016 - 23:52

Solo con ejemplo lo que pasó en mi instituto... Todos participamos, las enviamos o nos reimos, Crea morbo y tal, luego ya lo que me parece mal es que por sea tía pues la tengamos que insultar a la chavala que encima no tiene culpa de nada.

por jaretaldea9 el Lun 10 Oct 2016 - 16:25

Lo de la Uni si que me enteré... se lio bastante gorda. Creo que son casos que cada vez se están dando mas, por ejemplo en mi instituto paso que una chica le paso a su novio en pelotas y este luego las paso... la verdad es que la chavala sufrio bastante porque lo tpico, no?? La llamaron puta zorra y guarra, e imagino que en esos casos también pasaría un poco igual

Tanto en los grupos como en los foros, destaca la presencia y la importancia que adquiere la sexualidad -y sexualizaciónfemenina. De este modo, los videos y fotos sexuales son elementos que circulan cotidianamente, especialmente entre los grupos de WhatsApp de los chicos, como algunos de los consultados relatan:

por axi10 el Jue 13 Oct 2016 - 00:19

Si suele ser muy común que nos lleguen videos de estos estilos... Y fotos... Abre el grupo del Whatsapp del equipo y tienes siempre alguno...

por pelilloscarmesi el Jue 29 Sep 2016 - 0:08

Todos conocemos casos parecidos, y en mi instituto ha pasado sobre una chica. Al final la pobre se tuvo que cambiar de instituto y todo, porque todo el mundo la insultaba por los pasillos y tal, y es que era un canteo veias que todo el mundo estábamos con el video viéndolo... En este caso, que se grabaron haciéndolo (como los jugadores del Eibar) y el chaval lo paso por ahí.. Ella quedo como la guarra, y el otro no era de mi institituto pero me comentaron que iba muy orgulloso enseñando el video...

Enviar y recibir este tipo de fotos se convierten en prácticas de reafirmación de la masculinidad, ya que muestran una potencia sexual que se concibe como uno de los elementos vertebradores de esta identidad (Martino y Pallota-Chiarolli, 2005: 99; Ringrose y Renold, 2010). Reírse y comentar estos videos online abre paso a nuevos escenarios de legitimación 
de la masculinidad hegemónica, de exaltación de la sexualidad masculina y denigración de la feminidad, que sustentan la asimetría de poderes.

\subsection{Culpabilización y revictimización de las chicas que sufren ciberacoso}

Se detecta un exceso de responsabilidad femenina respecto a su sexualidad y su cuerpo. Como puede observarse en los siguientes fragmentos, las chicas entienden que su sexualidad queda construida por parámetros desiguales y perciben que pueden ser "víctimas constantes" (Megias y Ballesteros, 2014; De Beauvouir, 1949). Esta asunción hace que se responsabilicen de evitar este tipo de situaciones, entendiendo que aquellas que no lo hacen "algo andan buscando" o "han sido tontas". Como muestra se destacan los siguientes extractos ilustrativos:

por JGarrigo el Mier 12 Oct 2016 - 12:56

Veo que es algo que suele pasar lo de enviar fotos... si no quieres que se pasen fotos tuyas, no las envíes, y asi se evitan un monton de problemas... porque esque sino la otra explicación que me queda es que estas buscanco esto... por Opino como todos de todo el Vie 14 Oct 2016 - 00:23

Yo también pienso que es mas con el tema de de cuidado con lo que envias... es que yo creo que muchas veces pasan estas cosas porque la chica ha sido verdaderamente tonta, por confiar en alguien que no merecia, deberíamos andar con mas cuidado, y protegernos mas

Por tanto, las adolescentes que son (ciber)acosadas, bien por parte de las chicas, bien por parte de los chicos, son sancionadas socialmente y sufren una revictimización. Así, como diría Lagarde (1990), el cuerpo pasa a ser de dominio público, un cuerpo erótico, y se reproducen procesos normativos del sistema patriarcal que condenan y oprimen la sexualidad y las experiencias sexuales femeninas.

\section{Objetivización de las corporalidades femeninas}

Tal y como se señalaba en los grupos de discusión, las corporalidades femeninas de las adolescentes consultadas adquieren un peso relevante en la definición de sus autoconceptos, a la vez que se dibujan como espacios de dominación masculina. En ese sentido, se considera importante visibilizar las agresiones sexistas que han sufrido las participantes en los foros. Estas chicas verbalizan una vivencia de expropiación de sus cuerpos, alimentadas por la mirada externa y temerosas del juicio ajeno. En sus palabras se detecta sufrimiento y angustia:

por Hellokitty el Sáb 1 Oct 2016 - 13:16

Claramente le damos importancia aunque no queramos.. porque siempre te acaba viniendo a la cabeza.. y te acabas rayando y pensandolo, en mi caso lo más ofensivo ha sido con mi fisico y le di mucha importancia, por 1327 el Sáb 30 Abr 2016 - 12:49

Por ask te mandan comentarios en plan: enseñame de las tetas, o que talla de sujetador usas? O quieren quedar contigo... o peores cosas... Sobre todo cuando se meten con tu cuerpo, y te empiezan a poner puntuaciones, es 
asqueroso... Y, sí, lo pasas muy mal, poque al final crees que eres ese número o que te pasa algo a tu cuerpo, y no paras de mirarte y sentirte mal contigo misma... Es mejor no hacer caso, pero al final lo haces

Los chicos, por su parte, confirman la existencia de estas agresiones, de las que han sido testigos o participantes activos. Algunos de ellos señalan que en los portales internacionales en los que se habla sobre videojuegos, las chicas son más insultadas y denigradas por su físico, y reciben comentarios sexistas. Otros relatan que han comprobado estos hechos al hacerse pasar por chicas en algunos chat internacionales, tal y como nos cuenta un joven:

por The Mannis el Jue 27 Oct 2016 - 10:33

A ver lo ves y te ríes y con los colegas te metes y alguna vez también te haces pasar por una tía porque hay montón de degenerados.

por axi10 el Jue 27 Oct 2016 - 10:40

Yo me he metido con los colegas un par de veces pero siempre para echarte unas risas... También nos hemos hecho pasar por tías porque siempre hay alguno que te habla... y te mofas de él pensando que eres una tia y luego le eneseñas la caras de todos.

por ianu el Dom 2 Oct 2016 - 18:38

Por ejemplo en twitch, que es como una tele pero en internet, la gente que retransmite las partidas de los videojuegos que juega cuanto mas vamos y mejor seas mas seguidores tienes, pero en el caso de las mujeres, cuanto mas grandes los pechos y mas canalillo enseñan mas gente las ve.

En otras palabras, las chicas se adentran en este ámbito desde una posición inferior y con el riesgo de ser insultadas y denigradas (Gobierno Vasco, 2013; INJUVE, 2011). Su cuerpo y sexualidad se convierten en elementos de esclavitud, y opresión (De Beauvoir, 1949). Esto es, la vida online y offline son mundos que se interconectan e intercambian estructuras, reflejándose mutuamente, aumentando, generando otras...

En definitiva, la Red se convierte en un espacio desigual y asimétrico, que configura posturas jerarquizadas. Mientras que los chicos heterosexuales ocupan la cúspide de la pirámide -ocupando posiciones más elevadas, cuanto mayor alarde hacen de su virilidad y potencia sexual-, en los márgenes se sitúan aquellas feminidades recalcadas (Connell, 1995) que guardan con recelo su sexualidad. En una posición inferior encontraríamos las masculinidades homosexuales, y en un escalón aún más bajo estarían "las otras", aquellas que han roto su pacto con el secretismo sexual. De esta manera, la posición propia se sustenta en el rechazo de aquellas y aquellos que se sitúan en escalones más bajos, y sobre todo de aquellas que ocupan los escalones inferiores, para mantener el orden de género.

\section{Conclusiones}

Este artículo recoge los principales resultados obtenidos tras la realización de grupos de discusión y foros telemáticos con adolescentes con el objetivo de analizar las diferentes formas de acoso sexual y sexista ejercidas en las redes sociales y TRIC. Se ha pretendido profundizar en la persistencia de las estructuras patriarcales machistas y sexistas en el mundo online, reflejo ampliado del mundo offline, y conocer cuáles son algunas de las consecuencias de las ciberviolencias ejercidas 
contra las chicas y contra los adolescentes que no responden a la heteronormatividad. La investigación es exploratoria y no admite generalizaciones, pero los resultados obtenidos permiten constatar como tendencias: un mayor acoso sexual y sexista en las redes hacia las mujeres y un paralelismo entre las formas de violencia machista y las ciberviolencias. En estas violencias se aprecian relaciones de poder, posiciones asimétricas y masculinidades hegemónicas y heteronormativas, desde las que se legitiman y normalizan determinados comportamientos y violencias.

Para visibilizar y desmontar estas formas de agresión en las redes es importante llevar a cabo un análisis con perspectiva de género, que atienda e identifique las diferentes formas de la violencia de género y permita entender la fundamentación y el origen las formas de acoso, y, así poder visibilizar los riesgos inherentes y constatados en esta investigación, que presentamos a continuación:

- Los resultados del estudio nos sitúan ante un panorama desigual, en el que las chicas consultadas, se sienten y se perciben como presas y cautivas del machismo, y los chicos promueven estas situaciones desde "la diversión". Las chicas se adentran en este espacio desde una posición inferior (impuesta) y con el riesgo de ser insultadas y denigradas (Gobierno Vasco, 2013; INJUVE, 2011). Los resultados muestran que existe un encarcelamiento simbólico-cultural de la sexualidad femenina; las adolescentes consultadas se sienten y se hacen esclavas de su propia sexualidad. Se atribuye una responsabilidad individual a la víctima (ámbito privado) sin percibir que es un problema social (ámbito público).

- Se observa la normalización de determinados contenidos pornográficos hetero-dirigidos, sobre todo por parte de los chicos, como herramientas auto-didácticas y como referentes para valorar y proyectar su propia sexualidad.

- Se constata que la exposición por parte de las chicas de una sexualidad libre, supone su despersonalización y su cosificación como objeto erótico (De Beauvoir, 1949; Lagarde, 1990; Megias y Ballesteros, 2014). En la exposición de relaciones sexuales de una pareja heterosexual, el grupo solo penaliza y estigmatiza a la chica a través de acusaciones, reproches y juicios que trascienden a formas de acoso también en el mundo offline. Los cuerpos de las chicas se convierten en territorios de dominación y las ciberviolencias contra ellas suponen un ejercicio de control y de mantenimiento del orden social de género. Esa objetivación de las corporalidades femeninas, que son evaluadas y tratadas obviando su humanidad, incide en la valoración que las chicas hacen de sí mismas y en sus niveles de confianza.

- Existen ciberviolencias contra personas que subvierten la heteronormatividad. Se han encontrado otras formas de ciberacoso sufridas por las identidades no-normativas, y que precisan de atención y análisis, por lo que es necesario hablar de (ciber)violencias machistas, para poder aglutinar las diferentes formas de agresión que sufren tanto las mujeres (por el hecho de serlo) como las personas que rompen la normatividad.

La investigación ha permitido visibilizar y analizar cómo las chicas y chicos adolescentes utilizan las redes sociales y cuál es su respuesta ante los casos de ciberviolencias machistas y cómo los interpretan. No queremos finalizar esta última reflexión, sin mencionar que existen investigaciones que reconocen que en el uso de las redes sociales también se generan ámbitos reivindicativos y sumamente potentes para la transformación social e identitaria (Haraway, 1991; Plant, 1998; Turkle, 1997; Zafra, 2005b). Las redes sociales y las TRIC no deben comprenderse únicamente como elementos dañinos y reproductores de esquemas tradicionales, sino también como herramientas estratégicas para combatir estas formas de ciberviolencia. 
El ciberacoso sexual y/o sexista contra las adolescentes. Nuevas versiones online de la opresión...

\section{Referencias bibliográficas}

Alcañiz, M. (2001). Cambio tecnológico y género: planteamiento y propuestas. Asparkía. Investigació Feminista, (12), 19-33. Doi: http://dx.doi.org/10.6035/Asparkia.

Bartrina, M. J. (2014). Conductas de ciberacoso en niños y adolescentes. Hay una salida con la educación y la conciencia social. Educar, 50(2), pp. 383-400. Doi: doi.org/10.5565/rev/educar.672

Boix, M., Fraga, C. y Sedón, V. (2001). El viaje de las internautas. Una mirada de género a las nuevas tecnologías. Madrid, España: PardeDÓS.

Bourdieu, P. (1999). La dominación masculina. Barcelona, España: Anagrama.

Buelga, S. y Pons, J. (2011). Agresiones entre adolescentes a través del teléfono móvil y de internet. Psychosocial Intervention, 21 (1), pp. 91-101. Doi: doi.org/10.5093/in2012v21n1a2

Buelga, S., Cava, M. J. y Musitu, G. (2010). Cyberbullying: victimización entre adolescentes a través del teléfono móvil y de Internet. Psicothema, 22 (4), pp. 784-789. ISSN 0214-9915.

Castaño, C. (2005). Las mujeres y las tecnologías de la información. Madrid, España: Alianza.

_ (2008). La segunda brecha digital. Madrid, España: Feminismos Editorial.

Castaño, C. y Caprile, M. (2010a). Marco conceptual sobre género y tecnología. En C. Castaño, y M. Castells (Ed.), Género y TIC. Presencia, posición y políticas (pp. 21-81). Barcelona, España: UOC Ediciones.

Castells, M. (2005). La era de la Información. Madrid, España: Alianza.

Chriss, M. y Stewart, F. (2000). Internet Communication and Qualitative Research. A handbook for Researching Online. New Delhi, India: Sage Publications.

Connell, R. (1995). Masculinidades. México D.C, México: PUEG. Universidad nacional autónoma de México.

Crouh, M. A. (2001). Thinking Sexual Harassment . A guide for the perplexed. New York, EEUU: Oxford University Press.

De Beauvoir, S. (1949): El segundo sexo. Feminismos. Valencia, España: Ed. Cátedra. Universitat de Valencia. Instituto de la mujer.

Del Rey, R., Felipe, P. y Ortega-Ruiz, R. (2012). Bullying and cyberbullying: Overlapping and predictive value of the co-occurrence. Psicotherma, 24(4), pp. 608-613. ISSN 0214 - 9915.

Delegación del Gobierno para la Violencia de Género (2014). El ciberacoso como forma de ejercer la violencia de género en la juventud: Un riesgo en la sociedad de la información y el conocimiento. Madrid, España: Ministerio de Sanidad, Servicios Sociales e Igualdad.

Díaz, C. (2001). Las internautas de los institutos. “Chateo” y “navegación” como diferencias de género en la Red. Asparkía. Investigació Feminista, 12, pp. 53-65.

Egan, E. D. y Hawkes, G. L. (2012). Sexuality, youth and perils of endangered innocence: how history can help us get past the panic. Gender and Education, 24(3), pp. 269-284. Doi: doi.org/10.1080/09540253.2012.666232. 
European Institute for Gender Equality (EIGE) (2018). Gender equality and youth: the opportunities and risks of digitalisation. Lithuania: EIGE.

Frosh, S., Phoenix, A., y Pattman, R. (2002). Young Masculinities. New York, EE.UU.: Palgrave.

García, A., y Nuñez, S. (2008). Apuntes sobre la identidad virtual de género. En S. Nuñez, y E. Pérez (Ed.), La representación/ presencia de la mujer en los medios de comunicación, 11 (pp. 41-58). Alicante, España: Feminismo/s.

Gil, E. (2008). Representaciones sociales de la masculinidad y la feminidad. En R. Pérez-Amat, S. García, N. Puentes y A. García, Comunicación, identidad y género Vol. I (pp. 11-20). Madrid, España: Fragua.

Gobierno Vasco (2011). El acoso sexual y el acoso por razón de sexo en el trabajo. Vitoria- Gazteiz, España: Dirección de Atención a las Víctimas de Violencia de Género. Departamento de interior.

_ (2013). La desigualdad de género y el sexismo en las redes sociales. Una aproximación cualitativa al uso que hacen las redes sociales las y los jóvenes en la CAPV.Vitoria-Gasteiz, España: Departamento de Educación.

Haraway, D. J. (1991). Ciencia, cyborgs y mujeres. La rebelión de la naturaleza. Madrid, España: Feminismos.

Hesse-Biber, S. y Leavy, P. (2011). The Practice of Qualitative Research. United States of America: Sage Publications.

Hooley, T., Marriott, J. y Wellens, J. (2013). What is online research? Using the Internet for Social Science Research. London, United Kingdom: Bloomsbury Academic.

Instituto de la Mujer (2008a). Mujeres y nuevas tecnologías de la información y la comunicación. Madrid, España: Instituto de la Mujer.

INJUVE (2011). Cifras y jóvenes. Sondeo de opinión. TIC y jóvenes. Madrid, España: Observatorio Juventud en España.

Jiménez-Albiar, M. I., Piqueras, J. A., Mateu-Martínez, O., Carballo, J. L., Orgilés, M. y Espada, J. P. (2012). Diferencias de sexo, característica de personalidad y afrontamiento uso de Internet, el móvil y los videojuegos en la adolescencia. Health and Addictions / Salud y Drogas, 12(1), pp. 61-82. ISSN: 1578-5319.

Lagarde, M. (1990). Los cautiverios de las mujeres. Madresposas, monjas, putas, presas y locas. Madrid, España: horas y HORAS.

Lenhart, A. (2009). Teen and Sexting. Pew Research Center.Washington D.C, EE.UU.: Pew Internet y American Life Project.

Martino, W., y Pallota-Chiarolli, M. (2005). Being Normal is the only way to be. Adolescents perspectives on gender and school. Sydney, Australia: University of South Wales.

Megias, I., y Ballesteros, J. C. (2014). Jóvenes y género. Estado de la Cuestión. Madrid, España: Centro Reina Sofía.

Mitchell, K. J., Jones, L. M., Turner, H. A., Shattuck, A. y Wolak, J. (2016). The Role of Technology in Peer Harassment: Does It Amplify Harm for Youth? Pychology of violence, 6(2), pp. 193-204. Doi: doi.org/10.1037/a0039317.

Mora, B. (2004). Del acoso sexista a la sexualización del acoso sexual. Apuntes para una lectura en clave feminista. Mujeres en la red. Periódico feminista. Disponible en Internet formato pdf: http://www.mujeresenred.net/IMG/article_PDF/article_a50.pdf (Consultado el 11-02-2013). 
El ciberacoso sexual y/o sexista contra las adolescentes. Nuevas versiones online de la opresión...

Navarro, R. (2016). Gender Issues and Cyberbullying in Children and Adolescents: From Gender Differences to Gender Identity Measures. En R. Navarrro, S. Yubero y E. Larrañaga (Eds.), Cyberbullying Across the Globe. Gender, Family, and Mental Health (pp. 35-44), Cuenca, España: UCM.

Osborne, R. (2001). La violencia contra las mujeres. Realidad social y políticas públicas. Madrid: UNED.

_ (2009). Apuntes sobre violencia de género. Barcelona: Ediciones Bellaterra, Barcelona.

Philips, L. (2013). A Multi-method Examination of Race, Class, Gender, Sexual Orientation, and Motivations for Participation in the YouTube-based "It Gets Better Project". Chapel-Hill, EE.UU.: UMT.

Pineda, S., y Aliño, M. (1999). El concepto de la adolescencia. En F. Cruz, S. Pineda, N. Martínez y M. Aliño, Manual de prácticas clínicas para la atención integral a la Salud en adolescencia (pp. 15-23). Ciudad de la Habana, Cuba: MINSAP.

Plant, S. Ceros+ Unos. (1998). Mujeres digitales+ la nueva tecnocultura. Barcelona, España: Ciencias Sociales/ Destino.

Paasonen, S. (2011) Revisiting cyberfeminism. Communications 36, pp. 335-352. Doi: 10.1515/COMM.2011.017

Powell, A. y Henry, N. (2014). Blurred Lines? Responding to 'Sexting' and Gender-based Violence among Young People. Children Australia, 39, pp. 119-124. Doi: doi.org/10.1017/cha.2014.9.

Renold, E. (2002). Presumed: (Hetero) sexual, heterosexist and homophobic harassment among primary school girls and boys. Childhood, 9(4), pp. 415-434. Doi: 10.1177/0907568202009004004.

_ (2007). Girls, Boys and Junior Sexualities. London, United Kingdom: Taylor y Francis.

Ringrose, J., y Renold, E. (2010). Normative cruelties and gender deviants: the performative effects of bully discourses for girls and boys in school. British Educational Research Journal, 36(4), pp. 573-596. Doi: doi.org/10.1080/01411920903018117. Rovira, M. (2001). Los códigos de género en la adolescencia. En A. Tomé y X. Rambla, Contra el sexismo. Coeducación y democracia en la escuela (pp. 39-56). Madrid, España: Síntesis, S.A.

Ruiz, C. (2014). La construcción social de las relaciones amorosas y sexuales en la adolescencia. Graduando violencias cotidianas. Jaén, España: Diputación Provincial de Jaén.

Ruiz Olabuénaga, J. I. (2012): Teoría y práctica de la investigación cualitativa. Bilbao, España: Universidad de Deusto.

Strassberg, D. S., McKinnon, R. K., Sustaíta, M. A. y Rullo, J. (2012). Sexting by High School Students: An Exploratory and Descriptive Study. Archives of Sexual Behavior, 42, pp. 15-21. Doi: 10.1007/s10508-012-9969-8.

Taylor, S.J y Bogdan, R. (2000). Introducción a los métodos cualitativos. Barcelona, España: Paidós.

Tubert, S. (2008). La construcción de la identidad sexuada en la adolescencia. En Instituto de la Mujer, Imaginario cultural, construcción de identidades de género y violencia: formación para la igualdad en la adolescencia (pp. 50-88). Madrid, España: Ministerio de igualdad.

Turkle, S. (1997). La vida de la pantalla. La construcción de la identidad en la era de Internet. Barcelona: Paidós.

UNESCO (2017). School Violence and Bullying: Global Status Report. Sustainable Development Goals. UNESCO. 
Valdes, F. (2013). Unpacking Hetero-Patriarchy: Tracing the Conflation of Sex, Gender y Sexual Orientation to Its Origins. Yale Journal of Law y the Humanities, 8 (1), pp. 161-221.

Vázquez, N., Estébanez, I., y Herbón, M. (2013). Violencia Bella. El cuerpo adolescente como territorio de control. Análisis de vulnerabilidad y resistencia a las presiones sobre el autoconcepto y el cuerpo femenino entre las y los adolescentes de seis municipios de Bizkaia. Bilbao, España: Medicus Mundi.

Wajcman, J. (2004). El Tecnofeminismo. Madrid, España: Feminismos.

Weeks, J. (1993). El malestar de la sexualidad. Significados, mitos y sexualidades modernas. Talasa: Madrid

Zafra, R. (2005a). Las cartas rotas. Espacios de igualdad y feminización en Internet. Almería, España: Premio Ensayo "Carmen de Burgos “, 2000. Instituto de Estudios Almerienses.

_ (2005b). Netianas. N(H)acer mujer en Internet. Madrid, España: Lengua de Trapo S.L.

_ (2010). Un cuarto propio conectado. (Ciber)espacio y (auto)gestión del yo. Madrid, España: Fórcola.

Ley Orgánica 3/2007, de 22 de marzo, para la igualdad efectiva de mujeres y hombres. Disponible en la Red: https://www. boe.es/buscar/doc.php?id=BOE-A-2007-6115 (Consultado el 19-6-2015). 
